\title{
Drug Utilization Study in Ophthalmology Outpatients in a Tertiary Care Hospital
}

\author{
Hardik V Vaniya ${ }^{1, *}$, Nishita H Darji ${ }^{2}$, Vikas R Patel ${ }^{3}$, Devdatta J Gohel ${ }^{3}$ \\ ${ }^{1}$ Department of Pharmacology, M P Shah Government Medical College, Jamnagar, India \\ ${ }^{2}$ Department of Pharmacology, Smt. NHL Municipal Medical College, India \\ ${ }^{3}$ Department of Ophthalmology, M P Shah Government Medical College, India
}

Copyright $(2016$ by authors, all rights reserved. Authors agree that this article remains permanently open access under the terms of the Creative Commons Attribution License 4.0 International License

\begin{abstract}
Aims: The present study was carried out to describe the patterns of prescription and drug use in Ophthalmology outpatients at GG hospital, Jamnagar, Gujarat, India. Methods: Total of 600 patients were included during 3 months duration and average number of drugs per encounter, percentage of drugs prescribed by generic name, percentage of encounters with antibiotics prescribed, percentage of encounters with an injection prescribed, percentage of drugs prescribed from the WHO Essential Drug List 2015, number of fixed dose combinations prescribed and percentage of antimicrobial agents prescribed were analyzed. Results: Prescription analysis showed that the average number of drugs per prescription was $3.20 \%$. Eye drops were the most commonly prescribed $(66.8 \%)$ dosage form, followed by ointment (18.4\%), capsules $(8.66 \%)$ and tablets $(6 \%)$. Dosage forms were recorded in $99.2 \%$ drugs, information about the frequency of drug administration was present in $96 \%$ of the drugs and duration of therapy was recorded in $84.7 \%$ of the drugs prescribed. The drug classes that were used most frequently were Antibiotics (45\%), Ocular lubricants (24.7\%), NSAIDS (17.4\%) and Anti-allergy medications (8.1\%). Conclusion: Common prescription errors were minimum. However polypharmacy was common.
\end{abstract}

Keywords Drug Utilization Study, Ophthalmology, Outpatients, Polypharmacy

\section{Introduction}

The World Health Organization (WHO) has defined drug utilization research as the marketing, distribution, prescription, and use of drugs in a society, with special emphasis on the resulting medical, social and economic consequences.[1] Drug utilization studies are good tools to ascertain the role of drugs in the society. They provide a socio-medical and economic basis for making healthcare decisions. [2]

Drug therapy is an important component of patient care management in health care settings. Many pharmaceutical products are available in the market often having unaffordable cost. [3] Irrational use of drugs and dosage forms result in potential adverse effects to the patients and cause financial burden. To avoid such problems, every member of the healthcare system should practice rational use of drugs.

Important criteria for rational drug use are accurate diagnosis, proper prescribing, correct dispensing, suitable packing and patient adherence. [4] To improve the overall drug use, especially in developing countries, international agencies like World Health Organization (WHO) have given standard drug use indicators. [5] These indicators help doctors to know the shortcomings in prescription writing and allow to improve performance from time to time.

Recently in Ophthalmology, there have been many drug developments and introduction of new ocular therapeutic agents.[6,7] The efficacy and safety of drugs used in ophthalmology is often confounded by the accuracy of dose administration of the ophthalmic preparation by the patients which in turn is dependent on proper education by the Ophthalmologist, a rational prescription and proper comprehension of the information provided to the patient, besides many other factors.[8] Indiscriminate use of topical ophthalmic non-steroidal anti-inflammatory drugs and antibiotics can result in many adverse effects like local irritant effects, histological and structural changes in conjunctiva, indolent corneal ulcers and even severe systemic effects such as exacerbation of bronchial asthma. $[9,10]$ In order to improve drugs therapeutic efficacy, minimize adverse effects, and delay development of resistance, drug utilization trends and patterns need to be evaluated periodically. [11]

Although rational use of drugs is quite a usual practice, monitoring of prescriptions and drug utilization studies can identify the associated problems and provide feedback to prescribers. Developing countries have limited resources for 
healthcare and drugs and it becomes very important to prescribe drug rationally so that the available resources can be utilized optimally. Present study will give insight about the prevalence of various ophthalmic diseases and drug utilization pattern and current prescribing practices of the ophthalmologists of Guru Gobindsingh Government Hospital, Jamnagar which is a tertiary care centre located in the city of Jamnagar and serves urban population of the city as well as the rural population which is referred from primary and secondary centres from the nearby villages. The outpatient care is a walk in care facility which provides all type of care to the patients. There is also an inpatient department for indoor admission for the patients.

\section{Materials and Methods}

\subsection{Study Design \& Method}

This was an open label, prospective, non-interventional, observational study conducted by Department Of Pharmacology in association with Department Of Ophthalmology, Shri M.P. Shah Medical College \& Guru Gobind Singh Hospital, Jamnagar. Diagnosis and line of treatment were decided by the Ophthalmologist, Department of ophthalmology, G. G. Hospital. No additional drugs or investigations were advised by us during study period. The study was conducted from between August 2015 to October 2015 after approval from ethics committee. Consecutive patients attending morning Ophthalmology OPD of G. G. Hospital Jamnagar were included in our study and from these, patients presenting with refractive errors, patients managed non-pharmacologically and patients who declined to provide consent were excluded. Data like age, sex, diagnosis, ongoing treatment were recorded from case record of patients. Total of 600 patients were included and informed consent of the patient for the study was taken. Identity of patients was kept confidential.

\subsection{Data analysis}

\subsubsection{WHO core Drug Use Indicators [1]}

Average number of drugs per encounter, percentage of drugs prescribed by generic name, percentage of encounters with antibiotics prescribed, percentage of encounters with an injection prescribed, percentage of drugs prescribed from the WHO Essential Drug List 2015[12] were analyzed.

\subsubsection{Additional Indices}

Number of fixed dose combinations prescribed and percentage of antimicrobial agents prescribed were analyzed.

\subsubsection{Statistical Analysis}

Descriptive statistics were performed. Data was entered and analyzed with Microsoft Excel 2013. Values were expressed as actual numbers, percentage and mean.

\subsection{Ethical considerations}

The study was commenced after obtaining approval from institutional ethics committee.

\section{Results}

Total 600 prescriptions were analyzed for study and total number of drugs in 600 prescriptions was 1925 . Number of drugs per prescription varied from one to five with average of 3.20 [Table 1]. The mean age of the study sample was $40.1 \pm 22.2$ years. $53.16 \%$ of the patients were females.

Table 1. Number of drugs prescribed per prescription

\begin{tabular}{|c|c|}
\hline $\begin{array}{c}\text { Number of drugs per } \\
\text { prescription }\end{array}$ & Number of prescriptions n (\%) \\
\hline One & $23(4 \%)$ \\
\hline Two & $263(44 \%)$ \\
\hline Three & $81(13 \%)$ \\
\hline Four & $32(5 \%)$ \\
\hline Five & $201(34 \%)$ \\
\hline Total & $600(100 \%)$ \\
\hline
\end{tabular}

Majority of the patients attending ophthalmology OPD had eyelid diseases (34\%), foreign body in eye (22\%), conjunctivitis $(20.3 \%)$ or trauma $(9.8 \%)$. [Figure 1] For these diseases, drugs were prescribed in five different dosage forms. [Table 2] Eye drops were the most commonly prescribed $(66.8 \%)$ dosage form, followed by ointment $(18.4 \%)$, capsules $(8.66 \%)$ and tablets $(6 \%)$.

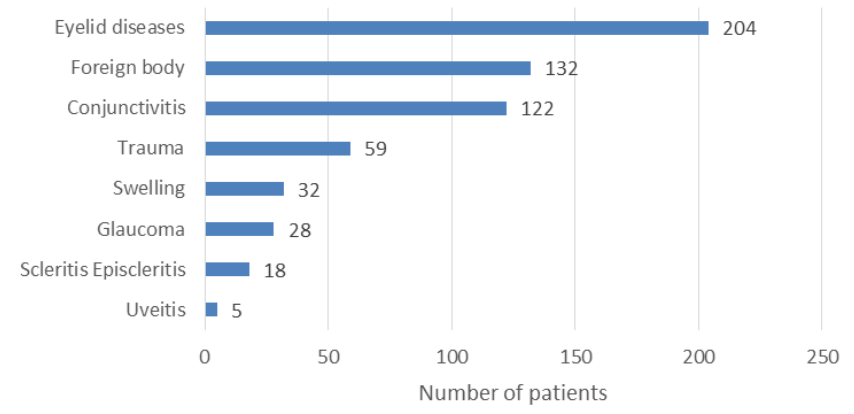

Figure 1. Morbidity pattern

Table 2. Dosage forms of drugs used

\begin{tabular}{|c|c|}
\hline Dosage forms & Number of drugs prescribed $\mathrm{n}(\%)$ \\
\hline Drops & $1286(66.8 \%)$ \\
\hline Ointment & $354(18.4 \%)$ \\
\hline Capsule & $166(8.6 \%)$ \\
\hline Tablet & $115(6 \%)$ \\
\hline Parenteral & $4(0.2 \%)$ \\
\hline
\end{tabular}

Study also revealed that the drugs were prescribed both by generic name $(42.6 \%)$ and brand name $(57.4 \%)$. The percentage of drugs prescribed from essential drug list was 
24\% (462/1925). [Table 3]

Dosage forms were recorded in $99.2 \%$ drugs, information about the frequency of drug administration was present in $96 \%$ of the drugs and duration of therapy was recorded in $84.7 \%$ of the drugs prescribed.

The most frequently drug class that were used were Antibiotics (45\%), Ocular lubricants (24.7\%), NSAIDS $(17.4 \%)$ and Antiallergy medications (8.1\%). [Figure 2]

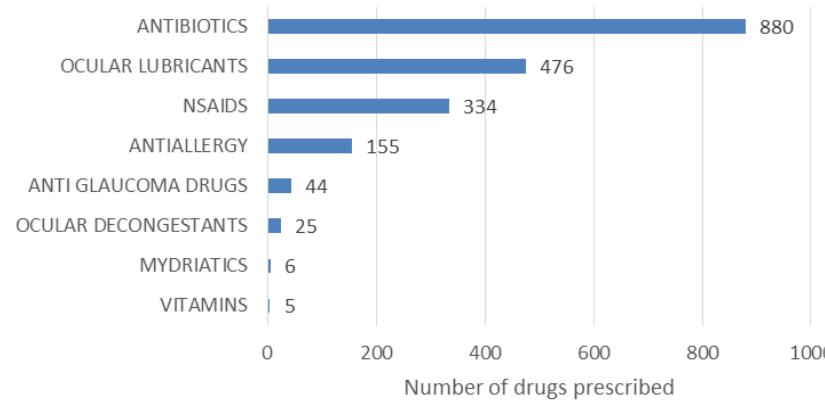

Figure 2. Drugs used

Table 3. Drug utilization based indicators

\begin{tabular}{|c|c|}
\hline Indicators assessed & Data value \\
\hline Average number of drugs per encounter & 3.2 \\
\hline Percentage of drugs prescribed by generic name & $42.6 \%$ \\
\hline Percentage of encounters with an antibiotic prescribed & $62.2 \%$ \\
\hline Percentage of encounters with an injection prescribed & $0.7 \%$ \\
\hline $\begin{array}{c}\text { Percentage of drugs prescribed from } \\
\text { WHO essential drug list }\end{array}$ & $24 \%$ \\
\hline Dosage forms recorded & $99.2 \%$ \\
\hline Frequency of therapy recorded & $96 \%$ \\
\hline Duration of therapy recorded & $84.7 \%$ \\
\hline
\end{tabular}

Among the prescriptions 240 drugs were prescribed as fixed dose combinations (FDC) that accounted for $12.46 \%$ of the total drugs prescribed. Commonly used FDCs were Chloramphenicol + polymyxin $\mathrm{B}$, Moxifloxacin + Dexamethasone, Gatifloxacin + dexamethasone, Tobramycin + Flourometholone, Brimonidine + Timolol. FDC are commonly prescribed for Glaucoma patients and allergic conditions of eye. Antibiotics and lubricating eye drops are commonly prescribed in non FDC polypharmacy.

The number of encounters with antibiotics was 373/600 $(62.2 \%)$ which constituted $45.7 \%(880 / 1925)$ of the total number of drugs prescribed. Antibiotics were the most commonly prescribed drugs either as single antibiotic or fixed-dose combination (FDC) with nonsteroidal anti-inflammatory drugs and FDC-antibiotic combination with steroids. Fluoroquinolones were the most commonly prescribed antimicrobial class of which ciprofloxacin, ofloxacin and moxifloxacin were the most frequently prescribed among the fluoroquinolones.[Table 4]

Antiallergy medications commonly used were Olapatidine, Sodium cromoglycate, Steroids like Flourometholone, and NSAIDSs like Ketorolac, Flurbiprofen.
Subgroup analysis of prescription patterns stratified by morbidity pattern is shown in Table 5. For Eyelid diseases Antibiotic drops, antibiotic ointments, lubricating drops and oral antibiotics were commonly prescribed. For Foreign body in eye and conjunctivitis usually antibiotic drops and lubricant drops were prescribed. For Trauma patients local and oral antibiotics were commonly prescribed drugs. Commonly used antiglaucoma drugs were Timolol, Brimonidine, Latanoprost, Pilocarpine, Acetazolamide, Mannitol and Glycerol. For treatment of Scleritis and Episcleritis antibiotic drops, lubricating drops, Flurbiprofen drops and oral NSAIDs were prescribed. For Uveitis Steroids and topical atropine were prescribed commonly.

Table 4. Antimicrobial drug utilization pattern

\begin{tabular}{|c|c|c|}
\hline $\begin{array}{c}\text { Dosage form } \\
\mathrm{N}(\%)\end{array}$ & Major Antibiotic & $\begin{array}{c}\text { Number of times } \\
\text { prescribed n (\%) }\end{array}$ \\
\hline & Ciprofloxacin & $194(32.94 \%)$ \\
Orops & Ofloxacin & $146(24.79 \%)$ \\
$589 / 880$ & Moxifloxacin & $73(12.39 \%)$ \\
$(66.93 \%)$ & Tobramycin & $58(9.85 \%)$ \\
& Azithromycin & $47(7.98 \%)$ \\
& Natamycin & $39(6.62 \%)$ \\
Ointment & Fluconazole & $29(4.92 \%)$ \\
$162 / 880$ & Moxifloxacin & $79(48.77 \%)$ \\
$(18.41 \%)$ & Chloramphenicol & $51(31.48 \%)$ \\
& Azithromycin & $20(12.35 \%)$ \\
Oral & Acyclovir & $10(6.17 \%)$ \\
$129 / 880$ & Ciprofloxacin & $60(37.04 \%)$ \\
$(14.66 \%)$ & Doxycycline & $38(23.46 \%)$ \\
& Amoxicillin+ & $29(17.90 \%)$ \\
\hline
\end{tabular}

Table 5. Subgroup analysis of prescription patterns

\begin{tabular}{|c|c|c|c|}
\hline Morbidity & $\begin{array}{c}\text { No. of } \\
\text { prescriptions }\end{array}$ & $\begin{array}{c}\text { No of drugs } \\
\text { prescribed }\end{array}$ & $\begin{array}{c}\text { No of drugs } \\
\text { prescribed/ } \\
\text { prescription }\end{array}$ \\
\hline Eyelid diseases & 204 & 961 & 4.7 \\
\hline Foreign body & 132 & 274 & 2.1 \\
\hline Conjunctivitis & 122 & 253 & 2.1 \\
\hline Trauma & 59 & 223 & 3.8 \\
\hline Swelling & 32 & 110 & 3.4 \\
\hline Glaucoma & 28 & 43 & 1.5 \\
\hline Scleritis/episcleritis & 18 & 52 & 2.9 \\
\hline Uveitis & 5 & 9 & 1.8 \\
\hline
\end{tabular}

\section{Discussion}

By conducting Drug utilization studies we obtain data about the patterns and quality of use, the determinants of drug use, and the outcomes of use. The WHO drug use indicators are highly standardized and are recommended for inclusion in drug utilization studies. [13] Our study was an attempt to describe the ophthalmic drug prescribing pattern in a tertiary care teaching hospital in Western India. The WHO core drug use indicators were used to primarily describe the drug use, particularly the prescribing indicators. 
The indicators of prescribing practices measure the performance of health care providers in several key dimensions related to the appropriate use of drugs.

Of the 600 prescriptions containing 1925 drugs studied, number of drugs per prescription ranged from one to five. Average number of drugs per prescription was $3.2 \%$. Other hospital based studies in India also reported 1.49-3.03 drugs per prescription. [14-18] This serves as a measure of degree of polypharmacy. It is preferable to keep the number of drugs per prescription as low as possible, to reduce the risk of adverse effects drug interactions, development of bacterial resistance and to decrease cost of therapy to the patient.[19] Most of the drugs have been prescribed topically, 1286 (66.8\%) in form of drops and 354 (18.4\%) in the form of ointment. Thus by giving the drugs topically for eye diseases has minimized their adverse effects.

In our study, prescriptions with generic name were just $42.6 \%$, which suggests popularity of brand names. The likely reason could have been the predictable response based on earlier clinical experience with a particular brand product and lack of the same confidence in generic drugs. Lack of confidence in generic drugs is not uncommon considering the evidence that in the absence of a strong regulatory control some of the marketed drug formulations might not be adhering to the standards prescribed.[20,21] Prescriptions of generic drugs could facilitate cheaper treatment for the patient.

The frequency of drug administration and drug therapy are the two most important parameters which, if not clearly recorded, can result in indiscriminate and injudicious use of drugs. The present study showed that the information about the frequency of drug administration was present in $96 \%$ of the drugs and duration of therapy was recorded in $84.7 \%$ of the drugs prescribed. In a small percentage of prescription without frequency or duration of therapy recorded usually Pharmacist told the patient dose and frequency. If there was any query by pharmacist patient was sent back to the ophthalmologist.

Antibiotics were frequent and number of encounters with antibiotics was $62.2 \%$. Antimicrobial agents constituted $45.7 \%(880 / 1925)$ of the total drugs prescribed and were the most commonly prescribed drugs either as single antibiotic or fixed-dose combination (FDC) with nonsteroidal anti-inflammatory drugs and FDC-antibiotic combination with steroids. Other similar studies in ophthalmology in India have reported $30 \%-45 \%$ encounters with antibiotics lower than our study [14-17]. Dry environment, poor education, less awareness and poor sanitation leads to more infective conditions which is represented by higher than average number of antibiotic usage. In our study most of antimicrobials were given topically as drops and ointment thus minimizing adverse effects.

Fluoroquinolones were the most common group of antibiotics prescribed which were similar to reports of previous studies done in ophthalmology [14-18]. Ciprofloxacin and ofloxacin were preferred in many prescriptions because they were available in the Hospital supply and patients could use them free of cost. Newer fluoroquinolones like Moxifloxacin were prescribed in severe cases.

There is a need to conduct many such studies in other departments as well, to audit large number of prescriptions and educate the prescribers on rational drug therapy for benefits and safety to the patient.

Limitation of the Study. It was a quantitative type of drug utilization study with the WHO/INRUD core prescribing indicators and therefore determining the quality of diagnosis and the appropriateness of drug choices was beyond the scope of prescribing indicators.

\section{Conclusions}

Polypharmacy was high in our study sample. Although the prescriptions were complete in terms of dose, duration and formulation, lack of use of generic names in the prescriptions needs to be addressed. High incidence of infective conditions necessitates consideration of the local drug sensitivity pattern and patient adherence to prescribed drug regimen.

\section{Conflict of Interest}

The authors have no funding sources or conflict of interest to report.

\section{Acknowledgements}

We are very grateful to faculty, resident doctors and staff of Department of ophthalmology, GG Hospital Jamnagar for their continuous support throughout the study period.

\section{REFERENCES}

[1] WHO, the selection of essential drugs. WHO technical report, 1977; 615: 36

[2] Bakssas I, Lunde PKM. National drug policies; the need for drug utilization studies. Trends Pharmacolo Sci 1986; 7:331.

[3] N. Y. Mirza, S. Desai, and B. Ganguly, "Prescribing pattern in a pediatric out-patient department in Gujarat," Bangladesh Journal of Pharmacology, vol. 4, no. 1, pp. 39-42, 2009.

[4] Nepalese National Formulary.1sted.Nepal: Kathmandu Nepal Ministry of Health;1997

[5] Maini Rajiv, Verma KK, Biswas NR et al. Drug utilization study in Dermatology in a tertiary hospital in Delhi. Ind J Physiol Pharmacol 2002; 46: 107-10.

[6] A. Duggirala, J. Joseph, S. Sharma, R. Nutheti, P. Garg, and T. Das, "Activity of newer fluoroquinolones against gram-positive and gram-negative bacteria isolated from 
ocular infections: an in vitro comparison," Indian Journal of Ophthalmology, vol. 55, no. 1, pp. 15-19, 2007.

[7] A. Leonardi, "Emerging drugs for ocular allergy," Expert Opinion on Emerging Drugs, vol. 10, no. 3, pp. 505-520, 2005.

[8] Tsai T, Robin AL, and Smith III JP. An Evaluation of How Glaucoma Patients Use Topical Medications: A Pilot Study. Transactions of the American Ophthalmological Society. 2007; 105: 29-35.

[9] Krishnaswamy K, Dinesh KB, Radhaiah G. A drug survey-precepts and practices. Eur J Clin Pharmacol 1985; 29: 363-70.

[10] Maini Rajiv, Verma KK, Biswas NR et al. Drug utilization study in Dermatology in a tertiary hospital in Delhi. Ind $\mathrm{J}$ Physiol Pharmacol 2002; 46: 107-10.

[11] K. Krishnaswamy, B. Dinesh Kumar, and G. Radhaiah, "A drug survey-precepts and practices," European Journal of Clinical Pharmacology, vol. 29, no. 3, pp. 363-370, 1985.

[12] 19th WHO Model list of Essential Medicines. Online available:http://www.who.int/medicines/publications/essenti almedicines/EML2015_8-May-15.pdf

[13] World Health Organization, Introduction to Drug Utilization Research, WHO, Oslo, Norway, 2003, http://apps.who.int/m edicinedocs/en/d/Js4876e/

[14] Jadhav PR, Moghe VV, Deshmukh YA. Drug utilization study in ophthalmology outpatients at a tertiary care teaching hospital. ISRN Pharmacol. 2013;2013:768792

[15] Meenakshi Nehru, K. Kohli, B. Kapoor, P. Sadhotra, V. Chopra, and R. Sharma. Drug utilization study in outpatient ophthalmology department of government medical college Jammu. JK Science, 7(3): 149-51, (2005).

[16] Yasmeen Maniyar Y, Bhixavatimath P, Akkone V. A drug utilization study in the Ophthalmology department of a medical college, Karnartaka. J Clin Diagn Res 2011; 5: 82-4.

[17] Biswas NR, Jindal S, Siddiquei MM et al. Patterns of prescription and drug use in ophthalmology in a tertiary hospital in Delhi. Br J Clin Pharmacol 2001; 51: 267-69.

[18] Rathnakar U P, Ashwin Kamath, Kashvi Gupta, Akash G, Sarah G Naushad, Mohamed Faheem Kotekar, Vignesh Shenoy. A Descriptive study of drug Utilization in Outpatients of Ophthalmology Department of a University Teaching Hospital in Southern India. J Clin Biomed Sci 2014; 4(4):346-49

[19] Sharma D, Reeta KH, Badyal DK et al .Antimicrobial prescribing pattern in an Indian tertiary hospital. Ind J Physio Pharmacol 1998; 42:533-37.

[20] Pickles V. Branded vs. Generic Ophthalmic Drugs: Balancing Cost and Care. Ophthalmology management. 2013; 7: 7-14.

[21] Weir RE, Zaidi FH, Charteris DG, Bunce C, Soltani M, Lovering AM. Variability in the content of Indian generic ciprofloxacin eye drops. Br J Ophthalmol. 2005; 89(9): 1094-6. 